\title{
The role of follow-up ultrasound and clinical parameters after abdominal MDCT in patients with multiple trauma
}

\author{
Lucas L Geyer, M Körner, U Linsenmaier, S Wirth, MF Reiser \\ and $\mathrm{T}$ Meind
}

\begin{abstract}
Background: Beside its value during the initial trauma work-up (focused assessment with sonography for trauma), ultrasound (US) is recommended for early follow-up examinations of the abdomen in multiple injured patients. However, multidetector CT (MDCT) has proven to reliably diagnose traumatic lesions of abdominal organs, to depict their extent, and to assess their clinical relevance.

Purpose: To evaluate the diagnostic impact of follow-up US studies after MDCT of the abdomen and to identify possible clinical parameters indicating the need of a follow-up US.

Material and Methods: During a 30-month period, patients with suspected multiple trauma were allocated. Patients with admission to the ICU, an initial abdominal MDCT scan, and an US follow-up examination after 6 and $24 \mathrm{~h}$ were included. Two patient cohorts were defined: patients with normal abdominal MDCT (group I), patients with traumarelated pathologic abdominal MDCT (group 2). In all patients, parameters indicating alteration of vital functions or hemorrhage within the first $24 \mathrm{~h}$ were obtained by reviewing the medical charts.

Results: Forty-four of 193 patients were included: 24 were categorized in group I (mean age, 4I.I years; range, 21-90 years), 20 in group 2 (mean age, 36.6 years; range, $16-7 \mid$ years). In group I, US did not provide new information compared to emergency MDCT. In group 2, there were no contradictory 6- and 24-h follow-up US findings. In patients with positive MDCT findings and alterations of clinical parameters, US did not detect progression of a previously diagnosed pathology or any late manifestation of such a lesion. In none of the patients with negative abdominal MDCT and pathological clinical parameters US indicated an abdominal injury.

Conclusion: Routine US follow-up does not yield additional information after abdominal trauma. In patients with MDCT-proven organ lesions, follow-up MDCT should be considered if indicated by abnormal clinical and/or laboratory findings.
\end{abstract}

\section{Keywords}

Multiple trauma, follow-up examination, whole-body computed tomography, ultrasound, clinical parameters

Date received: 20 June 2012; accepted: 29 June 2013

\section{Introduction}

Survival and long-term outcome of patients after multiple trauma improve with onset of adequate therapy within the "golden hour of shock" (1). A fast and thorough diagnostic and therapeutic algorithm is indispensable for optimal treatment results (2). The diagnostic impact of radiographs and ultrasound (US) is limited (3). Only the use of multidetector computed tomography (MDCT) allows a comprehensive diagnosis in
Institute for Clinical Radiology, Ludwig-Maximilians-University Hospital Munich, Munich, Germany

\section{Corresponding author:}

Lucas L Geyer, Institute for Clinical Radiology, Ludwig-MaximiliansUniversity Hospital Munich, Ziemssenstraße I, 80336 Munich, Germany. Email: lucas.geyer@med.uni-muenchen.de 
multiple trauma $(4,5)$. The incorporation of MDCT in the diagnostic workflow has been shown to significantly increase the probability of survival of the multiple injured patient (6).

As MDCT has proven to reliably diagnose traumatic lesions of abdominal organs, to depict their extent and to assess their clinical relevance (1), non-operative management of organ lesions in the hemodynamically stable patient recently became the preferred treatment option (7). Although success rates of non-operative management are high, concerns arose due to the possibility of initially missed abdominal injuries in MDCT, late onset of bleeding, and complications (8). Therefore, monitoring and surveillance of non-operatively treated patients remains challenging.

Besides its value during the initial trauma work-up (focused assessment with sonography for trauma), US is recommended for follow-up examinations of the abdomen in multiple injured patients according to the guidelines of the German Society of Trauma Surgery (9). In particular after admission to intensive care units (ICU), this US follow-up is performed to assess possible progression of organ lesions in patients with pathologic abdominal MDCT findings or to exclude late manifestations of lesions that were not reported on the MDCT scan (10-12). However, those algorithms were established in times of single-slice CT. With the advent of multidetector $\mathrm{CT}$ and its increased spatial resolution, impression over time has been that only few if any of these follow-up studies revealed clinically relevant information compared to the initial MDCT findings. Furthermore, a recent study has shown the involved high costs for follow-up US regarding staff and equipment (11).

The purpose of this retrospective study was to evaluate the diagnostic impact of follow-up US studies after MDCT of the abdomen and to identify possible clinical parameters indicating the need of a follow-up US.

\section{Material and Methods}

The design of this retrospective study was discussed with our institutional review board which indicated that neither board approval nor informed patient consent was required.

\section{Patients}

Based on the trauma registry of our institution, patients with admission to the trauma room in a time period of 30 months were analyzed. The hospital charts and discharge diagnoses as well as the imaging reports of these patients were reviewed by one of the authors. Out of this cohort, patients who (a) were admitted to the ICU, (b) underwent MDCT at the time of the arrival at the trauma room including abdominal scans, and (c) received at least two follow-up US examinations at 6 and $24 \mathrm{~h}$ after MDCT were included in the study.

The age and gender of the patients, the injury mechanism, the clinical parameters, including laboratory test results within the first $24 \mathrm{~h}$, and the radiological findings were recorded.

Exclusion criteria were incomplete US follow-up, discharge from the ICU, or death within $24 \mathrm{~h}$ after admission.

\section{Imaging technique}

All MDCT scans were obtained with a 4-row MDCT (Somatom Volume Zoom, Siemens, Erlangen, Germany). The MDCT scanner is integrated into our hospital's trauma room. Patients were scanned in supine position immediately after admission to the trauma room. Depending on the patients' general condition and hemodynamic stability, arms were placed aside the body, crossed at the thorax, or placed elevated above the head.

The trauma room standard imaging protocol of our institution consists of a whole body scan including a native scan of the head and a contrast-enhanced thoracic and abdominal scan (Table 1).

Images of the abdomen were acquired in the portovenous phase $75 \mathrm{~s}$ after injection of contrast media (300 mg of iodine $/ \mathrm{mL}, 2 \mathrm{~mL} / \mathrm{kg}$ body weight, injection rate $2.5-3.5 \mathrm{~mL} / \mathrm{s}$, no oral contrast). Scanning parameters for the abdominal scan were: collimation, $4 \times$ $2.5 \mathrm{~mm}$; effective slice thickness, $5.0 \mathrm{~mm}$; tube voltage, $120 \mathrm{kV}$; effective tube current-time product, $165 \mathrm{mAs}$.

All MDCT scans were initially reviewed on a Picture Archiving and Communication System (PACS) console (Impax 4.1, AGFA-Gevaert, Mortsel, Belgium) with two $2 \mathrm{k}$ high-resolution monitors and the reports were dictated immediately after scanning by the radiologist in charge with at least 3 years of experience. Findings were directly communicated to the head of the trauma team. The reports are validated within the next $12 \mathrm{~h}$ by a board licensed radiologist. Only these conclusive reports were retrieved for the study. In the final report, the injuries were graded according to the AAST (American Association for the Surgery of Trauma) injury scale, as recommended in literature (13). For this study, all CT images were re-evaluated by one author (MK) who is experienced in emergency radiology to confirm the grade of injury.

After admission to the ICU, abdominal US followup studies were routinely done 6 and $24 \mathrm{~h}$ after initial emergency MDCT. All US studies were performed bedside by means of a mobile sonography device (EnVisor C, Philips Medical Systems, Best, The Netherlands) using a $3.5 \mathrm{MHz}$ convex probe. US examinations were 
Table I. CT protocol for the standard whole-body CT.

\begin{tabular}{|c|c|c|c|c|}
\hline & Head & Chest & Abdomen & Spine (small FOV) \\
\hline \multicolumn{5}{|l|}{ Scan parameters } \\
\hline Tube voltage $(\mathrm{kV})$ & 120 & 120 & 120 & \\
\hline Tube current (effective mAs) & 210 & $140 *$ & $165^{*}$ & \\
\hline Collimation (mm) & $4 \times 1$ & $4 \times 2.5$ & $4 \times 2.5$ & \\
\hline Scan mode & Sequential & Helical & Helical & \\
\hline Table movement (mm) & 4 & 12.5 & 12.5 & \\
\hline \multicolumn{5}{|l|}{ Contrast medium injection } \\
\hline Contrast (mL) & $\varnothing$ & \multicolumn{2}{|c|}{$3 \mathrm{~mL} / \mathrm{s}$ injection rate } & \\
\hline Scan delay (s) & $\varnothing$ & 25 & 80 & \\
\hline \multicolumn{5}{|l|}{ Image reconstruction } \\
\hline \multicolumn{5}{|l|}{ Slice thickness (mm) (kernel) } \\
\hline Axial & 3 (brain), 2 (bone) & $\begin{array}{l}5 \text { (soft tissue), } \\
3 \text { (bone) }\end{array}$ & $\begin{array}{l}5 \text { (soft tissue), } \\
3 \text { (bone) }\end{array}$ & 3 (bone) \\
\hline Coronal & & $\begin{array}{l}3 \text { (soft tissue), } \\
3 \text { (bone) }\end{array}$ & 3 (soft tissue) & 3 (bone) \\
\hline Sagittal & & $\begin{array}{l}3 \text { (soft tissue), } \\
3 \text { (bone) }\end{array}$ & 3 (soft tissue) & 3 (bone) \\
\hline
\end{tabular}

*Dose modulation.

FOV, field of view.

performed by the radiologist in charge. B-mode imaging was primarily used; however, Doppler mode was used if deemed necessary by the performing radiologist. All radiologists in charge were board eligible or board certified and had at least 3 years of experience and at least 200 supervised US examinations as recommended by Scalea et al. (14).

\section{Clinical parameters}

For all patients, parameters which are routinely acquired within the first $24 \mathrm{~h}$ in the ICU were obtained by reviewing the medical charts. These data reflect the Acute Physiology Score which contributes to the APACHE (Acute Physiology and Chronic Health Evaluation) score, which is proven as prognostic score for critically-ill patients $(15,16)$. Parameters included: need for catecholamines, need for intubation, need for red blood cell transfusion, laboratory testing for hemoglobin and hematocrit, body temperature, mean arterial pressure, heart rate, breathing rate, sodium, and potassium. Laboratory parameter changes exceeding values given as normal by the institutional lab were considered as pathologic. Clinical parameters were taken from patients' ICU records at the time of US $24 \mathrm{~h}$ after trauma. The collected clinical and laboratory data indicate alteration of vital functions, hemorrhage (e.g. hemoglobin, hematocrit, mean arterial pressure, and heart rate), or a worsening of the patient's general condition, such as need for intubation (worsening respiratory function, hypoxia, increased breathing rate, etc.). For analysis, the trend over $24 \mathrm{~h}$ of each parameter was correlated with imaging data.

\section{Data analysis}

Abdominal MDCT and follow-up US findings concerning the location and extent of solid organ injuries and free abdominal fluid were correlated in all patients. Sensitivity, specificity, and positive and negative predictive values of US were calculated using MDCT findings as standard of reference. Differences in clinical parameters between patients with and without pathologic MDCT findings were evaluated using Fisher's exact test. The number of patients in whom clinical parameter alterations could be due to extra-abdominal injuries was assessed.

According to the abdominal MDCT findings two patient cohorts were defined:

- Group 1: patients with normal abdominal MDCT;

- Group 2: patients with trauma-related pathologic abdominal MDCT.

Pathologic abdominal MDCT findings were solid or hollow abdominal organic lesions, soft tissue pathologies which were definitely caused by the trauma (e.g. retroperitoneal hematoma) and free abdominal fluid. Solid organ injuries in CT and sonography were graded as described in current literature basically 
according to the AAST (American Association for the Surgery of Trauma) injury scale $(13,17,18)$. Other pathologic findings, such as hyper-attenuating kidney cysts, which were unlikely to be caused by trauma, were not rated. Therefore, the corresponding report was categorized as normal.

The Injury Severity Score (ISS) is a common scoring system to define multiple injured patients and was used for objective characterization of the patients' overall injury severity in this study. First, each injury was graded according to the Abbreviated Injury Scale (AIS) score. The AIS system ranks the injuries on a scale of 1 (minor) to 6 (unsurvivable) and takes six different body regions into account (head, face, chest, abdomen, extremities [including pelvis], external). Only the highest AIS score of the three most severely injured body regions had their score squared and added together to produce the ISS score, whereas the AIS of 6 was automatically assigned to an ISS of 75 points (maximum score) (19).

\section{Results}

\section{Demographic data}

During the study period, a total of 193 patients underwent abdominal MDCT in the trauma room. Fortyfour of them were admitted to the intensive care unit and received a 6- and 24-h US follow-up examination. The mean age of the patients was 42.3 years (range, 16-90 years; male:female ratio, 28:16). The mean ISS was $27 \pm 14$ (range, 9-64).

Injury patterns are displayed in Table 2 .

Twenty-four of the patients had a normal abdominal MDCT scan (group 1, $n=24$; mean age, 41.1 years; range, 21-90 years), 20 had pathologic abdominal

Table 2. Injury patterns in the patient collective.

\begin{tabular}{lr}
\hline Injury patterns & $n$ \\
\hline Bony fractures & 98 \\
Pneumothorax & 15 \\
Contusion of lung & 14 \\
Cerebral trauma & 12 \\
Lesion of spleen & 9 \\
Joint dislocation & 5 \\
Lesion of liver & 5 \\
Vascular lesion & 3 \\
Hemothorax & 2 \\
Lesion of the bladder & I \\
Lesion of pancreas & I \\
Lesion of adrenal glands & I \\
Rupture of diaphragm & I \\
\hline
\end{tabular}

findings (group 2, $n=20$; mean age, 36.6 years; range, 16-71 years). Twenty-one patients from group 1 and 18 patients from group 2 had additional extra-abdominal injuries.

\section{Ultrasound follow-up}

Concordance of $6 \mathrm{~h}$ and $24 \mathrm{~h}$ US follow-up studies was $100 \%$ in patients in group 1 . In these patients, US neither detected late manifestations of injury nor provided new information compared to emergency MDCT. In group 2, 6h US identified traces of free fluid which could not be detected in $24 \mathrm{~h}$ US in one patient. In two patients, $24 \mathrm{~h}$ US follow-up detected free fluid which was not described in $6 \mathrm{~h}$ follow-up US. In patients with organ lesions, there were no contradictory $6 \mathrm{~h}$ and $24 \mathrm{~h}$ follow-up US findings.

\section{Liver}

In five patients, liver lesions were detected by the emergency MDCT examination. In two cases the liver lesion could be identified by follow-up US (1 grade II and 1 grade III hematoma, blunt abdominal trauma), in three of them, the lesion was not detectable by US (1 grade III laceration, knife cut, 2 grade III hematoma, blunt abdominal trauma) (Fig. 1). In the remaining 39 patients without liver lesions, US reported no false-positive findings (sensitivity sonography $_{1}, 0.4$, specifity sonography, 1.0 , negative predictive value sonography, 0.93 , positive predictive value sonography $_{1.0}$ ).

\section{Spleen}

There were nine patients with splenic lacerations. In two patients, splenectomy was performed immediately after the initial MDCT because of grade V lesions (shattered spleen). In the remaining seven patients, the lesions could be monitored in three patients ( 1 grade II laceration, 1 grade II hematoma, 1 grade III laceration, blunt abdominal trauma), in four patients the lesions were not detected by follow-up US ( 2 grade I hematoma, 1 grade II hematoma, 1 grade II laceration, blunt abdominal trauma) (Figs. 2 and 3). In 38 patients without lesions of the spleen, US revealed no false-positive finding (sensitivity $_{\text {sonography }}, 0.43$; specifity $_{\text {sonography }}, 1.0$; negative predictive value $_{\text {sonography }}, 0.89$; positive predictive value $\left._{\text {sonography }}, 1.0\right)$.

\section{Free fluid}

Thirteen patients showed free intraperitoneal fluid on abdominal MDCT. In nine patients, this finding could be also detected in follow-up US. In four patients, free fluid was not identified by US. Thirty-one patients 

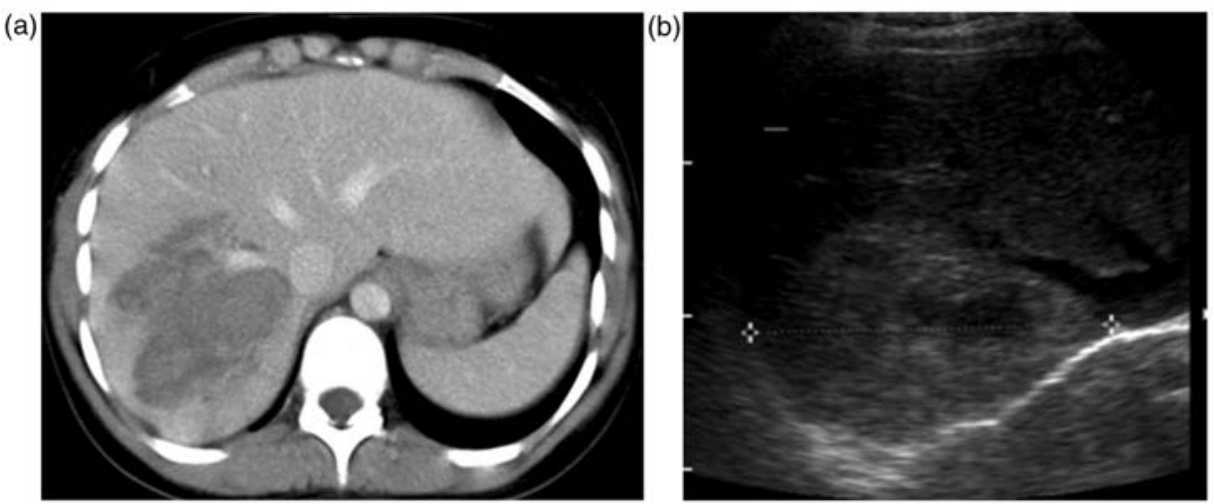

Fig. I. MDCT image of a 47-year-old man involved in a motor-vehicle accident. Emergency MDCT reveals a hypodense lesion of the right hepatic lobe corresponding to a grade III liver injury (a, axial contrast-enhanced MDCT, portovenous phase). Ultrasound confirmed the constant size of this lesion $6 \mathrm{~h}$ after ICU admission (b, ultrasound, axial orientation).
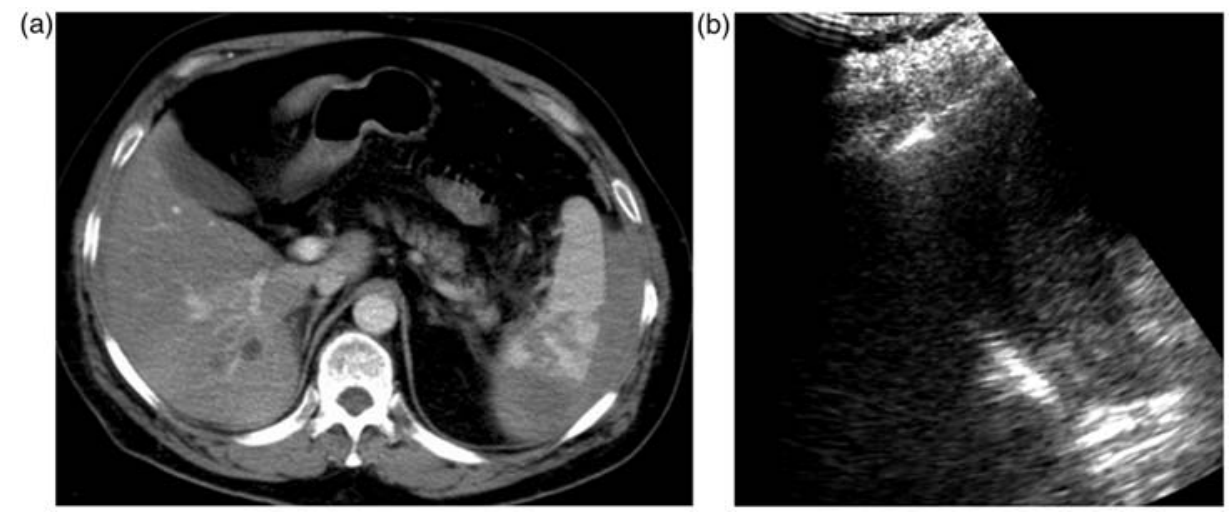

Fig. 2. A 36-year-old male construction worker suffering a left-sided blunt abdominal trauma at the construction site. MDCT shows a grade III laceration of the spleen with capsular tears and surrounding free fluid (a, axial contrast-enhanced MDCT, portovenous phase). These findings could be reproduced by follow-up ultrasound (b, ultrasound, sagittal orientation).

showed no free fluid in MDCT, which was confirmed in 28 of them by US. In three patients small amounts of free fluid were additionally diagnosed by follow-up US. In two of them, this finding was verified by a subse-

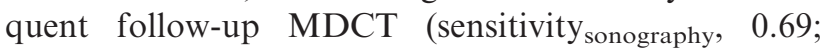
specifity $_{\text {sonography }}, 0.9$; negative predictive value $_{\text {sonog- }}$ raphy, 0.87 ; positive predictive value sonography $_{1}, 0.75$ ). However, even with MDCT, no injuries causing free abdominal fluid could be identified in these patients.

\section{Other lesions}

In three patients, lesions of the pancreas $(n=1)$, the adrenal gland $(n=1)$, and the urinary bladder lesion $(n=1)$ were present. None of these injuries could be identified in the follow-up US examinations.

\section{Correlation with clinical parameters}

In group 1, all but one patient had alterations of one or more clinical parameters. In group 2, all patients had at least one pathologic clinical parameter. Changes in clinical parameters did not significantly vary between patients with positive and negative abdominal MDCT findings (Table 3).

In none of the patients with negative abdominal MDCT and pathological clinical parameters US was indicative of an abdominal injury. In patients with positive MDCT findings and alterations of clinical parameters, US did not detect progression of a previously diagnosed pathology or any late manifestation of such a lesion.

According to these results, a follow-up algorithm for patients with multiple trauma after abdominal MDCT was proposed (Fig. 4).

\section{Discussion}

In this study, US was able to identify and follow-up organ injuries which were initially diagnosed by emergency MDCT in less than a half of the patients. A considerable amount of injuries could not be identified 


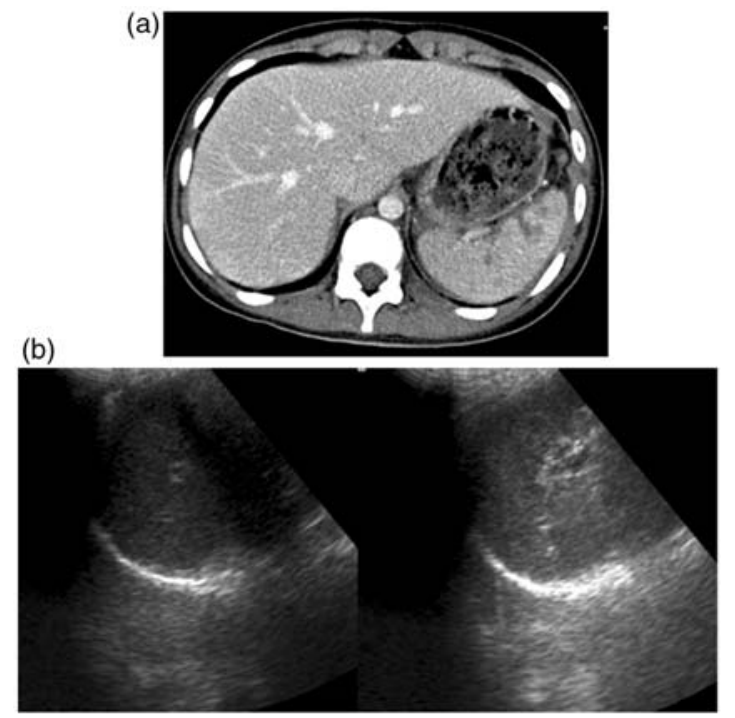

Fig. 3. A 50-year-old woman involved in a motor-vehicle accident as co-driver. MDCT detected small hypodense lesions of the spleen according to grade I lacerations; only small amounts of free fluid were found (a, axial contrast-enhanced MDCT, portovenous phase). Despite good ultrasound conditions, it was possible to depict neither the lesion nor free fluid (b, ultrasound, sagittal orientation).

Table 3. Evaluation of clinical and laboratory parameters with respect to abdominal MDCT results comparing the frequency of pathologic alterations between both groups.

\begin{tabular}{|c|c|c|c|c|}
\hline & \multicolumn{2}{|c|}{$\begin{array}{l}\text { Negative abdominal } \\
\text { MDCT (group I) }\end{array}$} & \multicolumn{2}{|c|}{$\begin{array}{l}\text { Positive abdominal } \\
\text { MDCT (group 2) }\end{array}$} \\
\hline & $n=24$ & $\%$ & $n=20$ & $\%$ \\
\hline \multicolumn{5}{|c|}{ Clinical parameters/indicators } \\
\hline Need of catecholamine & 15 & 62.5 & 12 & 60.0 \\
\hline Need of intubation & 16 & 66.7 & 14 & 70.0 \\
\hline Need of transfusion & 7 & 29.2 & 7 & 35.0 \\
\hline Temperature & 6 & 25.0 & 5 & 25.0 \\
\hline Mean arterial pressure & 11 & 45.8 & 17 & 85.0 \\
\hline Heart rate & 14 & 58.3 & 14 & 70.0 \\
\hline \multicolumn{5}{|l|}{ Laboratory parameters } \\
\hline Hemoglobin & 5 & 20.8 & 3 & 15.0 \\
\hline Hematocrit & 19 & 79.2 & 17 & 85.0 \\
\hline Sodium & 8 & 33.3 & 6 & 30.0 \\
\hline Potassium & I & 4.2 & I & 5.0 \\
\hline
\end{tabular}

There were no statistically significant differences in the number of altered parameters between group I and group 2 .

and monitored. This finding may reflect the well-known limitation of US in detecting organ lesions $(18,20,21)$. The low sensitivity of US may be due to suboptimal examination conditions at an ICU because of various medical devices such as central venous and/or arterial catheters and surgical dressings which may confine the examiner's field of view. However, in this study, the US operating radiologists were aware of the site of the trauma indicated by MDCT so that a focused examination should have become possible. These results resemble the findings of previous studies in which the ability of US to detect especially low-grade lesions of the liver, spleen, and kidney was shown to be limited $(12,20,22)$.

If free abdominal fluid was found in MDCT this finding was confirmed with US in most cases. In a number of patients, however, US could not verify this finding. In these patients notably only traces of free fluid were present at initial MDCT. In three patients, US detected free fluid, which was not seen on initial MDCT. Although these findings were confirmed by follow-up MDCT in two of these patients, their source could not be identified. In the other patient, the clinical condition was stable and no additional imaging was requested. Several studies have shown that the sensitivity of US for free intra-peritoneal fluid depends on the amount of fluid and the experience of the US operator $(23,24)$. In addition, the finding of free fluid needs to be interpreted in the context of several factors such as absolute amount of the fluid, physical examination, and hemoglobin value to ensure that decisions regarding the patient's management are based on the clinical presentation rather than on US findings alone. Isolated traces of free fluid can be safely managed conservatively and laparotomy is not mandatory in all cases (25).

In patients with negative abdominal MDCT findings, follow-up US led in no case to a new or inconsistent diagnosis. On the one hand, this could be due to the limited value of US to detect organ lesions. On the other hand, initial MDCT has a high diagnostic accuracy as well as a negative predictive value $(26,27)$. As a result, a follow-up MDCT examination or laparotomy was not required in any of these patients.

These results suggest that US appears to be an unreliable follow-up tool for organ lesions and free fluid at least in an ICU environment. However, for ethical and medico-legal reasons, follow-up algorithms including US as preliminary diagnostic tool in patients with organ lesions or free fluid as indicated by emergency MDCT may be maintainable. Nevertheless, the possibility of false-negative US findings has to be considered. In general, routine US added no new aspects to the initial abdominal MDCT findings which was probably due to the high sensitivity of MDCT and the low frequency of progressing abdominal injuries in this context. This is supported by a recent study which has also shown the low impact of follow-up US in multiple injured patients. In addition, the authors 


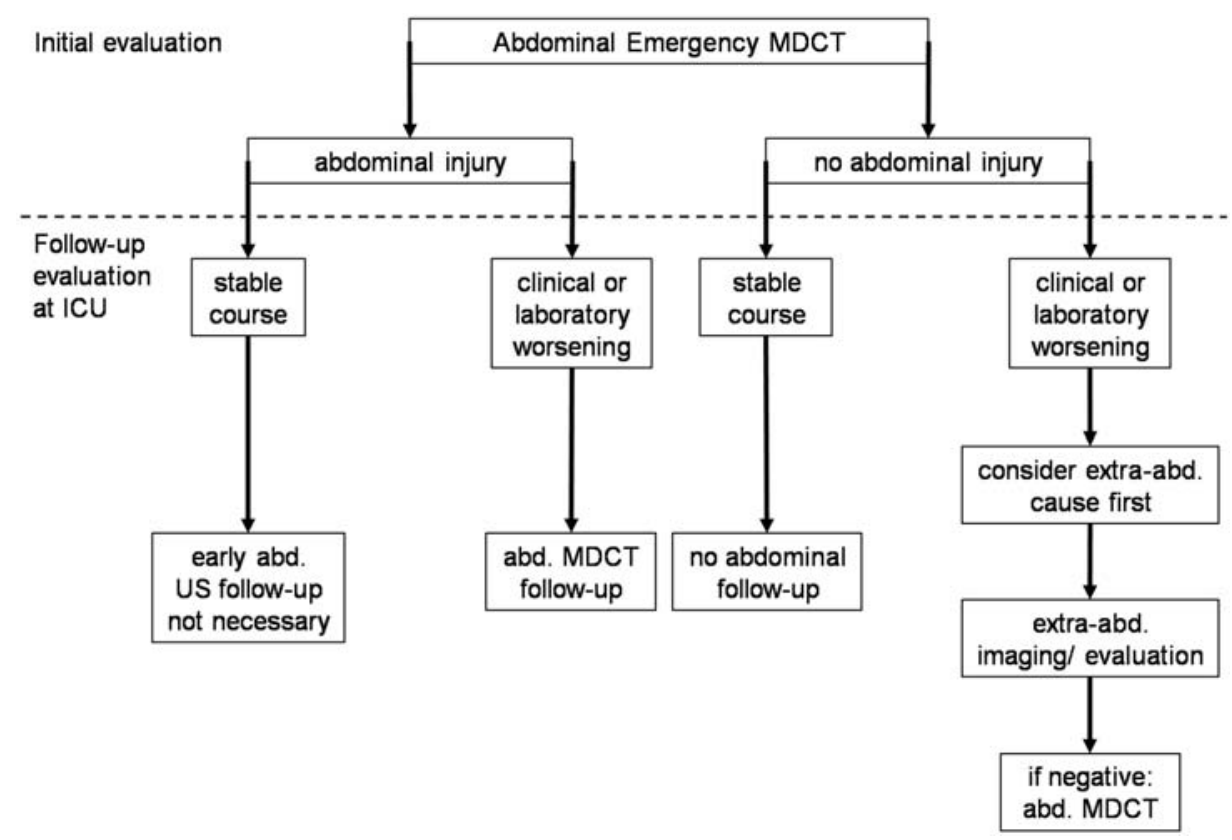

Fig. 4. Follow-up algorithm for patients sustaining multiple trauma, emergency MDCT, and admission to the ICU. In patients with stable course at ICU, early US follow-up is not necessary regardless of the presence or absence of abdominal injuries. In patients without proven abdominal injury but clinical or laboratory worsening conditions, at first an extra-abdominal reason should be excluded. If there is, no imaging studies of the abdomen are necessary unless physical situation deteriorates. In patients with known abdominal injury and worsening conditions or without identifiable reasons for clinical deterioration, ultrasound may be a fast imaging modality of first choice, as it is available at the ICU and may be operated as brief or focused survey by the physician in charge. However, one has to be aware, that there is a high probability of missing solid organ injuries. Therefore, fast transfer to the CT suite and MDCT follow-up is presumably the far better choice, if the patient's condition allows for it.

have highlighted the associated high costs (11). Therefore, routine US follow-up may be omitted in these patients.

The next aim of this study was to identify clinical and laboratory parameters which may predict the need for a follow-up US. Although all patients with positive MDCT findings of the abdomen had at least one pathologic parameter, their alteration was not indicative of an abdominal injury detected by US in both study groups. As mentioned above, all evaluated clinical and laboratory parameters are routinely obtained parameters which contribute to a continuous monitoring of ICU patients but are not specific for abdominal injuries (28). For instance, an extensive hemothorax can cause severe bleedings resulting in changes of clinical and laboratory parameters, such as decrease of blood pressure or increase of heart rate, or lung contusions can cause worsening of the respiratory function and require intubation. In the context of trauma patients, Rossaint et al. (29) have shown that a decrease of the hematocrit, central venous and central arterial pressure as well as an increase of heart rate are indicative of bleeding. In addition, Beck et al. (30) have identified that intubated patients are at risk for an abnormal abdominal CT after blunt trauma. Unfortunately, specific parameters predicting findings in imaging studies are hard to define. Therefore, intensive care management includes continuous and injury-related monitoring to identify the most likely cause of the patient's worsening condition as early as possible (28). However, the detailed process of priority-orientated decision-making which takes abdominal and extraabdominal injuries into account was not part of this study, because we have focused on the specific value of abdominal US in blunt trauma patients.

Limitations of this study arise from its retrospective design. Implication of an objective standard of reference was not possible. Repeated follow-up MDCT scans would have featured as good standard of reference for this study. However, due to the involved radiation exposure MDCT is not feasible for ethical reasons especially in patients with negative abdominal MDCT findings. Additionally, the value of follow-up MDCT has been questioned in recent studies $(31,32)$. Consequently, the clinical outcome had to serve as reference.

A further limitation might be the use of a 4-row MDCT scanner. This was unavoidable due to logistic reasons. Our scanner was dedicated to examine multiple injured patients and, therefore, installed within the trauma room. The advantage of this logistic concept is the undisturbed workflow for routinely scheduled patients, which could be scanned on a second 
CT system. On the one hand, an increased number of detector rows allow improving the spatial resolution in $\mathrm{z}$ axis, whereas the xy-plane resolution in axial slices can be considered as comparable. As axial slices are most important for image reading, the use of a CT system with more than four rows would only slightly increase the sensitivity for the detection of parenchymal injuries. However, high resolution multiplanar reconstructions are most beneficial in the detection of skeletal injuries and may improve the general diagnostic confidence. On the other hand, our study was focused on the detection of parenchymal lesions by US. A more sensitive reference method, e.g. 64-row MDCT, would lower the sensitivity of US even further. As a result, this would have a limited effect on our conclusion about the diagnostic value of US.

Furthermore, US is strongly investigator dependent, and the diagnostic sensitivity may be variable, when examinations are performed by different examiners. In this study, all radiologists were at least board eligible or already board certified to ensure a minimum standard of skills and good diagnostic validity.

The number of abdominal injuries in this study was small. However, with a positive abdominal MDCT in $11 \%$ of all our scanned patients, this number is comparable to previous data on prevalence of abdominal injuries. Thus, the patient population seems to be representative for a level I trauma center $(11,33)$.

In conclusion, the present study indicates that routine US exam at 6 and/or $24 \mathrm{~h}$ after MDCT of the abdomen does not yield additional information, both in patients with and without initial "normal" abdominal CT. Despite some advantages of US, such as use as a bedsite modality on an ICU, one has to be aware of potential false-negative results. Therefore, follow-up MDCT should be considered in patients with MDCT-proven organ lesions when indicated by clinical and/or laboratory findings because of a superior risk-benefit ratio.

\section{Funding}

This research received no specific grant from any funding agency in the public, commercial, or not-for-profit sectors.

\section{References}

1. Poletti PA, Wintermark M, Schnyder P, et al. Traumatic injuries: role of imaging in the management of the polytrauma victim (conservative expectation). Eur Radiol 2002;12:969-978.

2. Linsenmaier U, Krotz M, Hauser H, et al. Whole-body computed tomography in polytrauma: techniques and management. Eur Radiol 2002;12:1728-1740.

3. Geyer LL, Körner M, Reiser M, et al. Value of conventional radiography and ultrasound during initial treatment of traumatized patients. Notfall + Rettungsmedizin 2010;13:428-435.
4. Becker CD, Mentha G, Terrier F. Blunt abdominal trauma in adults: role of CT in the diagnosis and management of visceral injuries. Part 1: liver and spleen. Eur Radiol 1998;8:553-562.

5. Becker CD, Mentha G, Schmidlin F, et al. Blunt abdominal trauma in adults: role of $\mathrm{CT}$ in the diagnosis and management of visceral injuries. Part 2: Gastrointestinal tract and retroperitoneal organs. Eur Radiol 1998; 8:772-780.

6. Huber-Wagner S, Lefering R, Qvick LM, et al. Effect of whole-body CT during trauma resuscitation on survival: a retrospective, multicentre study. Lancet 2009; 373:1455-1461.

7. Gibson DE, Canfield CM, Levy PD. Selective nonoperative management of blunt abdominal trauma. J Emerg Med 2006;31:215-221.

8. Miller PR, Croce MA, Bee TK, et al. Associated injuries in blunt solid organ trauma: implications for missed injury in nonoperative management. J Trauma 2002; 53:238-242; discussion 42-44.

9. Stürmer KM, Dresing K, Bonnaire $\mathrm{M}$, et al. Recommended Guidelines for Diagnostics and Therapy in Trauma Surgery. Recommended Guidelines for Polytrauma Eur J Trauma 2001;27:137-150.

10. Blackbourne LH, Soffer D, McKenney $M$, et al. Secondary ultrasound examination increases the sensitivity of the FAST exam in blunt trauma. J Trauma 2004; 57:934-938.

11. Maurer MH, Winkler A, Wichlas F, et al. Costs and role of ultrasound follow-up of polytrauma patients after initial computed tomography. Rofo 2012;184:53-58.

12. Rozycki GS, Knudson MM, Shackford SR, et al. Surgeon-performed bedside organ assessment with sonography after trauma (BOAST): a pilot study from the WTA Multicenter Group. J Trauma 2005;59:1356-1364.

13. Miller LA, Shanmuganathan K. Multidetector CT evaluation of abdominal trauma. Radiol Clin North Am 2005;43:1079-1095.

14. Scalea TM, Rodriguez A, Chiu WC, et al. Focused Assessment with Sonography for Trauma (FAST): results from an international consensus conference. J Trauma 1999;46:466-472.

15. Knaus WA, Zimmerman JE, Wagner DP, et al. APACHE-acute physiology and chronic health evaluation: a physiologically based classification system. Crit Care Med 1981;9:591-597.

16. Knaus WA, Wagner DP, Draper EA, et al. The APACHE III prognostic system. Risk prediction of hospital mortality for critically ill hospitalized adults. Chest 1991;100:1619-1636.

17. Moore EE, Shackford SR, Pachter HL, et al. Organ injury scaling: spleen, liver, and kidney. J Trauma 1989;29:1664-1666.

18. Körner M, Krötz MM, Degenhart C, et al. Current role of emergency US in patients with major trauma. Radiographics 2008;28:225-242.

19. Baker SP, O'Neill B, Haddon W Jr, et al. The injury severity score: a method for describing patients with multiple injuries and evaluating emergency care. J Trauma 1974;14:187-196. 
20. Richards JR, Schleper NH, Woo BD, et al. Sonographic assessment of blunt abdominal trauma: a 4-year prospective study. J Clin Ultrasound 2002;30:59-67.

21. Stengel D, Bauwens K, Sehouli J, et al. Emergency ultrasound-based algorithms for diagnosing blunt abdominal trauma. Cochrane Database Syst Rev 2005;(2): CD004446.

22. Poletti PA, Kinkel K, Vermeulen B, et al. Blunt abdominal trauma: should US be used to detect both free fluid and organ injuries? Radiology 2003;227:95-103.

23. Gracias VH, Frankel HL, Gupta R, et al. Defining the learning curve for the Focused Abdominal Sonogram for Trauma (FAST) examination: implications for credentialing. Am Surg 2001;67:364-368.

24. Gracias VH, Frankel H, Gupta R, et al. The role of positive examinations in training for the focused assessment sonogram in trauma (FAST) examination. Am Surg 2002;68:1008-1011.

25. Brasel KJ, Olson CJ, Stafford RE, et al. Incidence and significance of free fluid on abdominal computed tomographic scan in blunt trauma. J Trauma 1998;44:889-892.

26. Ahvenjarvi L, Mattila L, Ojala R, et al. Value of multidetector computed tomography in assessing blunt multitrauma patients. Acta Radiol 2005;46:177-183.

27. Fang JF, Wong YC, Lin BC, et al. Usefulness of multidetector computed tomography for the initial assessment of blunt abdominal trauma patients. World J Surg 2006;30:176-182.

28. Waydhas C, Nast-Kolb D. Intensive care for patients with multiple injuries. Unfallchirurg 1999;102:474-491.

29. Roissaint C, Coats, Duranteau, Frenandez-Mondejar, Gordini, Stahel, Hunt, Neugebauer, Spahn. Key issues in advanced bleeding care in trauma. SHOCK 2006;26:322-331.

30. Beck D, Marley R, Salvator A, et al. Prospective study of the clinical predictors of a positive abdominal computed tomography in blunt trauma patients. J Trauma 2004;57:296-300.

31. Cox JC, Fabian TC, Maish GO 3rd, et al. Routine follow-up imaging is unnecessary in the management of blunt hepatic injury. J Trauma 2005;59:1175-1178; discussion 8-80.

32. Sharma OP, Oswanski MF, Singer D. Role of repeat computerized tomography in nonoperative management of solid organ trauma. Am Surg 2005;71:244-249.

33. Livingston DH, Lavery RF, Passannante MR, et al. Admission or observation is not necessary after a negative abdominal computed tomographic scan in patients with suspected blunt abdominal trauma: results of a prospective, multi-institutional trial. J Trauma 1998;44:273280; discussion 80-82. 